\section{АВТОРЕФЕРАТЫ ДИССЕРТАЦИЙ}

Текст на английском языке публикуется во второй части данного выпуска.

The text in English is published in the second part of the issue.

DOI: https://doi.org/10.30932/1992-3252-2021-19-4-18

Жайсан Иса. Обеспечение безопасности движения грузовых вагонов постройки КНР по железным дорогам стран Центральной Азии / Автореф. дис... канд. техн. наук. - М.: РУТ, 2021. - 24 c.

Цель данной диссертационной работы заключается в исследовании показателей динамики, безопасности движения и износа колеса и рельсов при наличии упругих скользунов постоянного контакта и диагональных тяг на сайлент-блоках, а также в определении диапазона рациональных значений параметров упругих скользунов и сайлентблоков.

Научная новизна работы заключается в разработке уточнённой, параметризированной математической модели движения грузовой тележки, оборудованного упругими скользунами постоянного контакта и диагональными тягами боковых рам по прямым и криволинейным участкам пути. Разработанная модель позволяет:

- проводить широкий спектр исследований динамических характеристик грузовых вагонов основных типов, оборудованных упругими скользунами постоянного контакта и диагональными тягами боковых рам при движении по участкам железнодорожного пути с произвольным очертанием с учётом воздействия неровностей рельсовых нитей;

- оценивать влияние отклонений в размеpax деталей вагона и износов отдельных элементов ходовых частей на динамические показатели и безопасность движения грузовых вагонов;

- оценивать влияние отклонений в техническом состоянии рельсовой колеи в прямых и криволинейных участках пути на динамические показатели грузового вагона;
- осуществлять подбор рациональных параметров геометрических, инерционных, жёсткостных и демпфирующих характеристик элементов грузового вагона и тележки.

В результате проведённых исследований произведена оценка влияния боковых скользунов постоянного контакта и диагональных тяг тележки 18-9996 на ходовые качества грузовых вагонов, безопасность движения, износ в системе колесо-рельс, произведён выбор рациональных величин параметров скользунов и сайлентблоков данного типа.

Разработана математическая модель движения грузовой тележки 18-9996, оборудованной боковыми опорами постоянного контакта и диагональными тягами, по прямым и кривым участкам пути. Графическая 3D-модель разработана в программном комплексе 3D-моделирования «КОМПAC3D». Модель была импортирована в программный комплекс «Универсальный механизм» и там была реализована в виде системы твёрдых тел, связанных посредством шарниров и силовых элементов.

Разработана полностью параметризованная, обобщённая расчётная схема и математическая модель бокового скользуна постоянного контакта, диагональных тяг, прокладки адаптера, адаптированная к изменению параметров и позволяющая моделировать различные варианты конструктивного исполнения и технического состояния опор и сайлент-блоков данного типа.

Разработанная математическая модель движения вагона обладает высокой степенью детализации и учёта реальной геометрии тел, что позволяет использовать её для изучения влияния параметров боковых опор кузова непрерывного контакта и диагональных тяг на динамику и безопасность движения подвижного состава с учётом возникающих в эксплуатации износов и повреждений.

Для определения угловой жёсткости проведены теоретические и экспериментальные исследования. Проведены расчёты для идентификации параметров расчётной модели диагональных связей тележек. Полученные результаты были близки к экспериментальным данным, что показывает адекватность выбранных параметров. 
Идентификация работы боковых опор кузова и диагональных тяг показала хорошую сходимость полученных результатов с результатами ранее проведённых исследований.

Сопоставление результатов компьютерного моделирования и экспериментальных данных по показателям динамики и безопасности движения показало их удовлетворительное совпадение. При этом расхождения не превышают 15-17 \%. Это свидетельствует о достоверности полученных результатов.

На основании результатов численного эксперимента и последующего анализа всех оцениваемых показателей определены рациональные величины прокладки адаптера, которые находятся в пределах: вертикальной жёсткости - 17-20•103 кН/м, продольной жёсткости 3,5-5•103 кН/М, поперечной жёсткости $-2 \cdot 103$ кН/м

Произведена оценка влияния упругодиссипативных характеристик сайлентблока диагональных тяг на показатели динамики, безопасности движения. Исходя из результатов компьютерного моделирования, в качестве рациональных значений жёсткости сайлентблоков, рекомендовано принимать значения в пределах от 5•103 Н/м до 7•103 H/M.

Результаты расчётов, выполненных для тележек, оборудованных скользунами постоянного контакта с роликами и диагональными тягами, с выбранными рациональными параметрами, показывают улучшение основных оцениваемых показателей при движении по прямолинейным и криволинейным в плане участкам пути.

Уменьшение износа в контакте колеса и рельса при наличии упругого скользуна с роликом и диагональных тяг боковых рам составляет $82 \%$ - для прямой в порожнем режиме, 36 \% - для прямой в гружёном режиме, $25 \%$ - для кривой $\mathrm{R}=650$ м в порожнем режиме, $30 \%$ - для кривой $\mathrm{R}=650$ м в гружёном режиме, $20 \%$ - для кривой $\mathrm{R}=350$ м в порожнем режиме и $36 \%$ - для кривой $\mathrm{R}=$ 350 м в гружёном режиме.

Расчёты по оценке влияния каткового механизма на оцениваемые показатели показали, что наличие ролика способствует снижению моментов сил трения между кузовом и надрессорной балкой при движении по кривой $\mathrm{R}=350$ м в среднем на $22 \%$, а умень- шение по основным показателям составляет 3-11\%.

05.22.07 - Подвижной состав железных дорог, тяга поездов и электрификация.

Работа выполнена и зашчищена в Российском университете транспорта.

Нгуен Суан Хиен. Методика определения требований к местам размещения комплексов фото-видеофиксации для условий Социалистической Республики Вьетнам / Автореф. дис... канд. техн. наук. - М.: МАДИ, 2021. - 23 с.

Целью диссертационного исследования является повышение безопасности дорожного движения в городе Ханой (Вьетнам) за счёт разработки методики определения требований к местам размещения комплексов фотовидеофиксации в заданных условиях финансирования.

Разработаны:

- математическая модель функционирования системы фотовидеофиксации, позволяющая дать оценку степени её влияния на характеристики транспортного потока и показатели аварийности;

- программа выбора структуры и обоснования параметров системы фото-видеофиксации нарушений правил дорожного движения;

- методика определения требований к местам размещения комплексов фотовидеофиксации для повышения эффективности их работы.

Проведён анализ показателей аварийности на автомобильном транспорте в мире и во Вьетнаме. Анализ показал, что в настоящее время ДТП являются социальными проблемами, с которыми сталкиваются во всём мире. Определена роль интеллектуальных транспортных систем в повышении безопасности и организации дорожного движения.

С целью повышения БДД и качества управления движением рассмотрен мировой опыт, который показал, что некоторые страны, такие как Швеция, Япония, Сингапур и др., получили хорошие результаты от реализации государственных программ повышения эффективности и безопасности дорожно-транспортного комплекса.

Анализ состояния применения интеллектуальных транспортных систем и системы 
фотовидеофиксации во Вьетнаме показывает, что практика внедрения ИТС во Вьетнаме имеет ряд существенных недостатков и проблем, таких как: отсутствие стандартов по установке и использованию комплексов фотовидеофиксации, отсутствие принципов синхронизации комплексов фотовидеофиксации в рамках системы; ограниченная функциональность комплексов фотовидеофиксации, главным образом для наблюдения за трафиком.

Обоснованы основные элементы целевой функции для оптимизации параметров установленного типа комплекса фотовидеофиксации, на основе следующих аспектов: повышения безопасности дорожного движения (за счёт уменьшения очагов аварийности) и эффективности организации дорожного движения (за счёт повышения пропускной способности); введения контроля на участках системных нарушений правил дорожного движения (нарушение скоростного режима, требований дорожной разметки, опасное и агрессивное вождение). Синтезированы 2 варианта целевой функции:

- определена максимальная эффективность установки системы ФВФ при ограниченном финансировании;

- определена максимальная приведённая эффективность установки системы ФВФ при максимальной рентабельности.

Разработана математическая модель влияния функционирования системы фотовидеофиксации на безопасность и на организацию дорожного движения. Такая модель способна определять дополнительные исходные данные с помощью оценок экспертов и метода анализа иерархий на основе имеющейся неполной информации официальных данных о ДТП во Вьетнаме.

Проведён анализа проблемных точек улично-дорожной сети города Ханой, в котором определены 10 точек с существующими камерами фотовидеофиксации и 10 точек без камер. На основе выявления суммарного взвешенного значения эффективности установки средств ФВФ определены точки, где следует устанавливать новые типы камер фотовидеофиксации.

Разработано программное обеспечение, предназначенное для оценки эффективности работы системы фотовидеофиксации. Программа проводит оценку эффективности работы системы фотовидеофиксации по установленному критерию. С помощью разработанного ПО обеспечивается выбор наилучшего варианта системы фотовидеофиксации для реализации её целей и задач.

Разработана методика выбора эффективного решения в создании системы фотовидеофиксации с учётом установленного критерия осуществления выбора её рационального варианта. Методика позволяет установить целесообразность использования различных технических средств системы фото-видеофиксации в рассматриваемых точках.

Разработана методика определения требований к местам размещения комплекса фотовидеофиксации во Вьетнаме в заданных условиях финансирования.

Дальнейшее развитие и совершенствование подхода настоящего исследования целесообразно реализовать по следующим направлениям:

- разработка системы национальных стандартов для ИТС в целом и для системы фотовидеофиксации, в том числе и при реализации научно обоснованных методов установления государственных приоритетов для внедрения системы фотовидеофиксации;

- разработка принципов координирования действий министерств и иных органов исполнительной власти для завершения системы сбора и хранения данных по безопасности дорожного движения, с целью повышения точности анализа и исследований в настоящей предметной области и применения достоверных методов расчёта и анализа;

- разработка программного обеспечения на основе современных языков, например $\mathrm{C}++$, Python, SQL, удобного в обновлении и выдаче результатов расчётов для пользователей, включая инвесторов.

05.22.10 - Эксплуатаџия автомобильного транспорта.

Работа выполнена и защищена в Московском автомобильно-дорожном государственном техническом университете (МАДИ).

Русских С. В. Нелинейная механика упругих трансформируемых и управляемых космических систем / Автореф. 
дис... докт. физ.-мат. наук. - М.: МАИ, 2021. -40 c.

Работа представляет собой вклад в решение актуальной и важной проблемы - создание надёжных математических моделей, позволяющих решать широкий класс задач нелинейной механики упругих трансформируемых и управляемых космических систем и конструкций, содержащих стержневые и тросовые элементы.

Получены новые уравнения динамики пространственного и плоского движения космического аппарата с выпускаемым тросом в центральном гравитационном поле Земли, которые могут быть использованы для численного моделирования движения космических аппаратов с тросовыми элементами, в частности - космического аппарата с выпускаемым из него тросом как космического буксира для отработавших свой ресурс спутников, захват крупных объектов космического мусора и т.д.

Впервые получены нелинейные уравнения с аналитическими выражениями всех коэффициентов для плоского движения в подвижной системе координат космического аппарата с присоединённой системой стержней, связанных между собой упруговязкими шарнирами, допускающими большие углы поворота. Эти уравнения позволяют решать новый класс задач развёртывания системы стержней из сложенного в пакет транспортировочного положения в рабочее положение различными способами, в том числе - за счёт инерционных сил вращения и передвижений космического аппарата.

Получены новые уравнения нестационарного поворота и нелинейных колебаний в плоскости крена космического аппарата с двумя упругими многосекционными панелями солнечных батарей. На основе этих уравнений можно проводить численную наземную отработку развёртывания панелей под действием предварительно напряжённых пружин в узлах, соединяющих секции.

Предложена функциональная схема и разработана математическая модель циклически симметричной антенны зонтичного типа, состоящей из гибких радиальных многозвенных стержней, связанных между собой в узлах по параллелям растяжимыми тросами. Разработан новый метод решения обратной нелинейной задачи квазистатического формообразования антенны после развёртывания радиальных стержней за счёт их изгиба под действием усилия, создаваемого демпфирующим гидроцилиндром с учётом реакций растяжимых тросов. Это будет способствовать созданию развёртываемых в космосе крупногабаритных антенн.

Впервые получены уточнённые уравнения термоупругих изгибных колебаний тонкостенного стержня-удлинителя с круговым поперечным сечением, соединённого с подвижным космическим аппаратом и подвергающегося солнечному нагреву с учётом изменений углов падения солнечных лучей за счёт поворотов элементов поверхности упругого стержня и с учётом внешнего и внутреннего теплоизлучения. Уравнения используются для расчёта нестационарных термоупругих колебаний стержня с космическим аппаратом при выходе его из тени.

Представлен подход получения уравнений движения упругих составных нелинейных систем с геометрическими связями на основании принципа возможных перемещений путём использования уравнений в связанных подвижных координатах и в обобщённых координатах для отдельных свободных подсистем с учётом неизвестных реакций взаимодействия и присоединения к этим уравнениям условий связи. Решение этих дифференциально-алгебраических нелинейных уравнений можно получить с помощью известных стандартных алгоритмов интегрирования для «жёстких» систем.

Разработан новый подход к решению терминальных задач пассивного силового и кинематического управления упругими, в общем случае нестационарными и нелинейными, системами с использованием метода Бубнова-Галеркина во временной области при их конечных передвижениях за определённое время из одного состояния (покоя или движения) в другое состояние с устранением колебаний в конце операции. Задачи для линейных систем с постоянными параметрами решаются путём разложения по собственным формам колебаний с использованием точных решений уравнений в нормальных координатах для нескольких низших форм, подлежащих устранению. При этом управляющее воздействие ищется в виде конечного ряда простых финитных функций с неизвестными коэффициентами, которые определяются из начальных и конечных условий. 
Предложен новый способ «настройки» нескольких низших собственных частот колебаний линейных систем с постоянными параметрами, многократно выполняющих однотипные операции, как быстродействующие манипуляторы, для устранения колебаний в конце каждой операции с использованием простой управляющей функции с одним неизвестным множителем.

\subsubsection{4 - Механика деформируемого} твёрдого тела.

Работа выполнена в Институте прикладной механики Российской академии наук (ИПРИМ РАН), зашищена в Московском авиационном институте (национальный исследовательский университет).

Сахаров Р. А. Техническое диагностирование профиля поверхности катания железнодорожных колёс в процессе эксплуатации / Автореф. дис... канд. техн. наук. - СПб.: ПГУПС, 2020. - 18 с.

Цель исследования - повышение безопасности движения поездов путём технического диагностирования критических состояний структурной неоднородности металла профиля поверхности катания и близлежащих слоёв цельнокатаных колёс грузовых вагонов (ЦКГВ) непосредственно в процессе эксплуатации (при движении поезда).

В результате выполнения диссертационной работы определено, что существующие системы технического диагностирования ЦКГВ на сети железных дорог из-за специфики их эксплуатации не могут обеспечить оценку структурной неоднородности металла ППК и близлежащих слоёв ЦКГВ во время эксплуатации, а, следовательно, и адекватную оценку остаточного технического ресурса.

Сформирован перечень требований к бортовому оборудованию, с помощью которого осуществляется техническое диагностирование ППК во время эксплуатации.

Разработаны методики оценки структурной неоднородности металла профиля поверхности катания и близлежащих слоёв ЦКГВ с возможностью определения их преддефектного состояния и прогнозирования остаточного технического ресурса на основе:

- усовершенствованного магнитовариационного метода контроля применительно к задачам технического диагностирования анизотропных объектов, например, железнодорожных колёс;

- результатов экспериментальных исследований по оценке структурной неоднородности ППК в условиях вагоноремонтного депо, лабораторий ПГУПС и ДЦНТИ, подтверждающих возможность качественной и количественной оценки структурного состояния металла ППК и близлежащих слоёв;

• полученных данных по индукции собственного магнитного поля ППК и близлежащих слоёв колёс, которые могут считаться критериями и параметрами основных дефектов, относящихся как к внезапному, так и постепенному механизму их образования и оказывающие наибольшее влияние на техническое состояние ППК и близлежащих слоёв ЦКГВ.

Разработано устройство технического диагностирования ППК и близлежащих слоёв ЦКГВ, позволяющее повысить точность регистрации дефектов, что связано с выявленной взаимосвязью магнитного и структурного состояния металла, благодаря чему было обосновано применение физических критериев для оценки конфигурации магнитного поля вблизи возможных зон концентрации напряжений ППК и близлежащих слоёв ЦКГВ.

Даны и обоснованы научно-технические предложения и рекомендации по применению разработанного устройства и методики оценки структурной неоднородности металла ППК и близлежащих слоёв ЦКГВ.

Подтверждённая экспериментально зависимость между значениями твёрдости ППК и близлежащих слоёв ЦКГВ и значениями индукции магнитного поля $B$ над ППК колеса показывает, что напряжения в приповерхностных слоях обода колеса и поверхность отклика сигнала магнитного поля $B$ коррелируют в плоскостях, соответствующих плоскостям скольжения кристаллической решётки структуры металла.

Основным экономическим эффектом является эффект от автоматизации процесса технического диагностирования в режиме реального времени. Показано, что техническое обслуживание и ремонт ВКП по фактическому состоянию по сравнению с традиционным позволяет значительно снизить эксплуатационные расходы. 
05.22.07 - Подвижной состав железных дорог, тяга поездов и электрификация.

Работа выполнена изашчщуена в Петербургском государственном университете путей сообщения Императора Александра I.

Степанова К. К. Совершенствование электротехнических устройств железнодорожного электроснабжения системы постоянного тока высокого напряжения / Автореф. дис... канд. техн. наук. - СПб.: ПГУПС, 2021. - 24 с.

Целью исследования является повышение производительности и энергоэффективности электрической тяги постоянного тока на основе увеличения уровня напряжения, применения достижений силовой электроники и цифровых технологий.

В диссертационной работе содержится решение научно-технической задачи, имеющей значение для совершенствования электротехнических устройств железнодорожного электроснабжения на основе системы постоянного тока высокого напряжения. Изложены научно обоснованные решения, которые позволяют повысить техникоэнергетическую эффективность системы электрической тяги, включающей подсистемы тягового электропривода, электрического подвижного состава и подсистемы тягового электроснабжения постоянного тока.

Предложена система электрической тяги постоянного тока высокого напряжения, позволяющая реализовать энергоэффективное и безопасное электрообеспечение высокопроизводительных систем движения поездов на железнодорожном транспорте. Установлено, что эффективность применения постоянного тока высокого напряжения по сравнению с системой переменного тока 25 кВ (2×25 кВ, 50 Гц) достигается за счёт повышения КПД контактной сети примерно на 6-8 \%, снижения расхода меди на сооружение контактной сети примерно на $20 \%$, упрощения контактной сети вследствие отсутствия нейтральных вставок; увеличения расстояния между тяговыми подстанциями и уменьшения их количества на электрифицированной линии; снижения отрицательного влияния на систему внешнего электроснабжения; отсутствия необходимости установки компенсирующих устройств реактивной мощности.
Установлен уровень высокого напряжения в контактной сети постоянного тока, при котором система постоянного тока по энергетической эффективности приближается к показателям системы однофазного переменного тока. Минимальным значением такого уровня является 18 кВ. Для повышения эффективности рекомендуется система постоянного тока 24 кВ и выше до 35 кВ в зависимости от условий применения системы.

Обоснована структура высоковольтного электротехнического комплекса тягового электроснабжения и электроподвижного состава постоянного тока высокого напряжения, включающая выпрямительный агрегат напряжением 24..35 кВ, электротяговые сети постоянного тока 24...35 кВ и входной преобразователь электроподвижного состава 24-35 кВ. Предложена структура преобразовательного комплекса на электроподвижном составе постоянного тока высокого напряжения на реверсивном преобразователе АИН ШИМ. На основе данной структуры преобразования электроэнергии на электроподвижном составе предложено разработать многосистемные ЭПС (24-35 кВ постоянного тока / 25 кВ 50 Гц / 3 кВ постоянного тока).

Разработана методика определения параметров устройств электроэнергетического комплекса системы электрической тяги постоянного тока высокого напряжения ещё на стадии проектирования жизненного цикла системы электрической тяги.

Построена имитационная модель системы постоянного тока с электроэнергетическим комплексом высокого напряжения и подтверждена его работоспособность путём проведения экспериментов на модели. Сформулировано предложение по реконструкции пассажиронапряжённых линий с переводом системы 3 кВ на централизованную систему тягового электроснабжением постоянным током высокого напряжения 24 кВ с универсальным электроподвижным составом.

05.09.03 - Электротехнические комплексы и системы.

Работа выполнена и зашищена в Петербургском государственном университете путей сообщения Императора Александра I. 